\title{
Development of a Dual-Porosity Model for Vapor-Dominated Fractured Geothermal Reservoirs Using a Semi-Analytical Fracture/Matrix. Interaction Term
}

\author{
Robert. W. Zimmerman, Teklu Hadgu, and Gudmundur S. Bodvarsson \\ Earth Sciences Division \\ Lawrence Berkeley Laboratory \\ University of California \\ Berkeley, California 94720
}

February 1993

This woik was supported by the Assistant Secretary for Conservation and Renewable Energy, Geothermal Division, of the U.S. Department of Energy under Contract No. DE-AC03-76SF00098. 


\section{DISCLAIMER}

This report was prepared as an account of work sponsored by an agency of the United States Government. Neither the United States Government nor any agency Thereof, nor any of their employees, makes any warranty, express or implied, or assumes any legal liability or responsibility for the accuracy, completeness, or usefulness of any information, apparatus, product, or process disclosed, or represents that its use would not infringe privately owned rights. Reference herein to any specific commercial product, process, or service by trade name, trademark, manufacturer, or otherwise does not necessarily constitute or imply its endorsement, recommendation, or favoring by the United States Government or any agency thereof. The views and opinions of authors expressed herein do not necessarily state or reflect those of the United States Government or any agency thereof. 


\section{DISCLAIMER}

Portions of this document may be illegible in electronic image products. Images are produced from the best available original document. 


\title{
DEVELOPMENT OF A DUAL-POROSITY MODEL FOR VAPOR-DOMINATED FRACTURED GEOTHERMAL RESERVOIRS USING A SEMI-ANALYTICAL FRACTURE/MATRIX INTERACTION TERM
}

\author{
Robert W. Zimmerman, Teklu Hadgu, and Gudmundur S. Bodvarsson \\ Earth Sciences Division, Lawrence Berkeley Laboratory \\ University of California, Berkeley, CA 94720
}

\begin{abstract}
A new type of dual-porosity model is being developed to simulate two-phase flow processes in fractured geothermal reservoirs. At this time it is assumed that the liquid phase in the matrix blocks remains immobile. By utilizing the effective compressibility of a two-phase water/steam mixture in a porous rock, flow within the matrix blocks can be modeled by a single diffusion equation. This equation in tum is replaced by a nonlinear ordinary differential equation that utilizes the mean pressure and mean saturation in the matrix blocks to calculate the rate of fluid flow between the matrix blocks and fractures. This equation has been incorporated into the numerical simulator TOUGH to serve as a source/sink term for computational gridblocks that represent the fracture system. The new method has been compared with solutions obtained using fullydiscretized matrix blocks, on a problem involving a three-dimensional vapor-dominated reservoir containing an injection and a production well, and has been found to be quite accurate.
\end{abstract}

\section{INTRODUCTION}

Although most geothermal reservoirs reside in fractured rocks, most models that have been developed to analyze their behavior have been based on porous medium approximations. In these models, the hydraulic behavior of the fractures and the matrix blocks are modeled together as a locally-homogeneous porous medium. It is weil-known, however, that porous medium models are poorly suited for predicting certain aspects of the behavior of geothermal wells, especially enthalpy transients, thennal front migration due to injection, and chemical traiser movement. Nevertheless, in many cases the porous rnedium approximation must be invoked, due to constraints $\mathrm{cf}$ computer time or cost. There is, consequently, a grea: need for improved numerical capabilities for the modeling of fractured geothermal reservoirs, using accurate and appropriate models.

In this paper we discuss our on-going research aimed at improved methods of simulating processes in fractured geothermal reservoirs. The main concept behind our approach is to analyze the heat and mass flow processes occurring within the matrix blocks by simplified equa- tions that enable us to avoid detailed discretization of the individual matrix blocks. These processes are generally govemed by diffusion-type partial differential equations. We model these diffusive processes with nonlinear ordinary differential equations that relate the average thermodynamic properties in the block to those at the outer boundary (in the fractures). The first stage of our work, dealing with isothermal flow of a singlephase fluid, was described at the 17th Stanford Workshop (Zimmerman et al., 1992). We have since extended the general approach to treat thermal conduction within the matrix blocks. This extension is straightforward, as shown by Pruess and Wu (1989), since the governing equation for conduction is exactly analogous to that for single-phase flow. In this paper we describe a further extension of this approach to processes involving two-phase conditions in which the liquid phase is immobile.

\section{DUAL-POROSITY MODELS}

We now briefly describe the main ideas behind dualporosity models for fractured reservoirs; for further details, see Barenblatt et al. (19/0), Warren and Root (1963), and Duguid and Lee (1977). In order to avoid a cumbersome notation, we will discuss these models with specific reference to single-phase, isothermal flow of a slightly-compressible fluid. Consider first a fracture network, and assume temporarily that the matrix blocks are impermeable. A basic assumption underlying the use of dual-porosity models is that there exists a scale at which it is possible to assign macroscopic properties to the fracture network, such as a permeability $k_{f}$, etc. Although it does not seem to be true that such a lengthscale always exists (cf., Long and Witherspoon, 1985), standard dual-porosity models assume that such a scale does exist. This scale then serves as a representative elementary volume (REV; see Bear, 1972) upon which a continuum formulation can be based. "Points" in the fracture network are then denoted by the vector $x_{f}$, where it is understood that properties defined at point $x_{f}$ are averaged over an REV centered at that point.

When a single-phase, slightly compressible fluid flows through such a fractured medium, the fluid pressure in the fractures is governed by the following equation (Matthews and Russell, 1967): 
$\phi_{f} \beta_{f} \frac{\partial P_{f}\left(x_{f}, t\right)}{\partial t}=\frac{k_{f}}{\mu} \nabla_{f}^{2} P_{f}\left(x_{f}, t\right)+Q\left(x_{f}, t\right)$,

where $\phi_{f}$ is the total fracture porosity, and $\beta_{f}$ is the total compressibility of the fractures and the fluid within them. The product $\phi_{f} \beta_{f}$ serves as the capacitance term in the diffusion-type equation (1). The pressure $P_{f}$ is the mean value of the fluid pressure in the fractures, averaged over some REV. The operator $\nabla_{f}^{2}$ is the Laplacian with respect to the coordinates $x_{f}$. The term $Q$ is a source/sink term representing the net volumetric addition of fluid to the fracture system, per unit of total volume. Although we assume here that the fracture continuum is isotropic, this assumption is not necessary.

Now assume that at each point $x_{f}$ in the fracture continuum, there is located a permeable matrix block of some specified shape. These matrix blocks can exchange fluid with the fracture continuum; hence we can identify the source/sink term $Q$ in equation (1) with the fluid exchange between the fractures and matrix blocks. Inside each matrix block the fluid pressure $P_{m}$ will, in general, vary from point to point. Points inside the matrix block are identified by two position variables: $x_{m}$ locites the point within the block relative to its centroid, and $x_{f}$ is used to locate that particular block within the fracture continuum. Fluid flow within each matrix block is governed by an equation analogous to (1):

$\emptyset_{m} \beta_{m} \frac{\partial P_{m}\left(x_{m}, t ; x_{f}\right)}{\partial t}=\frac{k_{m}}{\mu} \nabla_{m}^{2} P_{m}\left(x_{m}, t ; x_{f}\right)$.

The spatial derivatives are in this case taken with respect to the local variable $x_{m}$. The fracture/matrix interflow term $Q$ does not appear in equation (2) since, whereas the interfiow is assumed to be distributed throughout the fracture continuum as a source/sink term, the interflow enters the matrix blocks only at their boundaries. If the existence of a fracture skin is ignored (cf., Moench, 1984; deSwaan, 1990), then the pressure at the outer boundary of a given matrix block is equal to the local fracture pressure: i.e., if $x_{m}$ is on the boundary of the matrix block, then $P_{m}\left(x_{m}, t ; x_{f}\right)=P_{f}\left(x_{f}, t\right)$.

The system of equations (1) and (2) are coupled through the term $Q$, which can be found in principle by integrating the flux out of the boundary of each matrix block, $\partial V_{m}$, using Darcy's law (Duguid and Lee, 1977):

$Q\left(x_{f}, t\right)=\frac{-1}{V_{m}} \int_{\partial V_{m}} \frac{k_{m}}{\mu} \frac{\partial P_{m}}{\partial n} d A$,

where $n$ is measured along the outward unit normal to the boundary of the block. If the system of equations (1-3) were solved with a numerical simulator such as the integral-finite-difference simulator TOUGH (Pruess,
1987), the reservoir would be Hiscretized into a number of gridblocks, each representing a macroscopic region that contained a portion of the fracture continuum. Each of these regions would also therefore contain a certain number of matrix blocks, within which the flow will be governed by equation (2). For some problems the matrix blocks can be replaced, for computational purposes, by a single equivalent matrix block having the same volume and same fracture/matrix interface area as does the collection of actual matrix blocks. In order to accurately resolve the pressure gradients within the matrix blocks, this equivalent matrix block must be discretized into a number of concentric gridblocks. One efficient way of creating this type of dual-porosity grid is the MINC (Multiple INteracting Continua) method, which is described by Pruess and Narasimhan (1985). We have found that accurate MINC simulations over large time scales require roughly ten gridblocks in each equivalent matrix block. The total number of gridblocks used in a simulation will therefore be $11 N$, where $N$ is the number of fracture gridblocks. For threedimensional problems, this number will usually be impractically large, which suggests the desirability of replacing the fine-gridding in the matrix blocks with a readily-computed source/sink term.

\section{LUMPED-PARAMETER MODELS}

The earliest double-porosity models, developed by Barenblatt et al. (1960) and Warren and Root (1963), treated the matrix blocks in a lumped-parameter fashion. The equations of such an approach can be derived as follows. We first integrate equation (2) over an entire matrix block, and use the divergence theorem to convert the volume integral on the right into a surface integral, to find

$\phi_{m} \beta_{m} \frac{\partial \bar{P}_{m}\left(x_{f}, t\right)}{\partial t}=\frac{1}{V_{m}} \int_{\partial V_{m}} \frac{k_{m}}{\mu} \frac{\partial P_{m}}{\partial n} d A$,

where the average pressure $P_{m}$ is defined by

$\bar{P}_{m}\left(x_{f}, t\right)=\frac{1}{V_{m}} \int_{V_{m}} P_{m}\left(x_{m}, t ; x_{f}\right) d y$

Comparison of equations (3) and (4) shows that $P_{m}$ is governed by the following equation:

$\phi_{m} \beta_{m} \frac{d \bar{P}_{m}\left(x_{f}, t\right)}{d t}=-Q\left(x_{f}, t\right)$.

Equations (1) and (6) provide two equations for the three variables $P_{f}, \bar{P}_{m}$, and $Q$. To complete the system of equations, $Q$ must be expressed as a function of $P_{f}$ and $\bar{P}_{m}$. 
Barenblatt et al. (1960) and Warren and Root (1963) assumed that $Q$ is proportional to the difference between $P_{f}$ and $\bar{P}_{m}$, so that

$Q\left(x_{f}, t\right)=\frac{-\alpha k_{m}}{\mu}\left(P_{f}-\bar{P}_{m}\right)$

where $\alpha$ is a parameter that depends on block shape, and has dimensions of area $^{-1}$. The governing equation (6) for $\bar{P}_{m}$ then takes the form

$\phi_{m} \beta_{m} \frac{d \bar{P}_{m}\left(x_{f}, t\right)}{d t}=\frac{\alpha k_{m}}{\mu}\left(P_{f}-\bar{P}_{m}\right)$.

For a given shape of the matrix block, $\alpha$ can be chosen such that equation ( 8$)$ is asymptotically accurate at late times (see Zimmerman et al., 1992). For spherical matrix blocks of radius $a_{m}$, which will be used in this paper for illustrative purposes, $\alpha=\pi^{2} / a_{m}^{2}$.

The Warren-Root equation is known to be inaccurate in the early stages of diffusion into a matrix block (Streltsova, 1983; Dykhuizen, 1990). A more accurate interaction equation is that proposed by Vermeulen (1953):

$\phi_{m} \beta_{m} \frac{d \bar{P}_{m}}{d t}=\frac{\pi^{2} k_{m}\left[\left(P_{f}-P_{i}\right)^{2}-\left(\bar{P}_{m}-P_{i}\right)^{2}\right]}{2 \mu a_{m}^{2}\left(\bar{P}_{m}-P_{i}\right)}$,

where $P_{i}$ is the initial pressure in the matrix block. This interaction equation was shown to be fairly accurate for a wide range of boundary conditions (see Zimmerman et al., 1992). Recognizing that the combination $\pi^{2} k_{m} / \phi_{m} \beta_{m} \mu a_{m}^{2}$ is the product of the shape-factor $\alpha$ and the hydraulic diffusivity $D=k_{m} / \phi_{m} \mu \beta_{m}$, we see that equation (9) can be used for other diffusive processes, such as heat conduction.

\section{TWO-PHASES, IMMOBIE LIQUID}

Porous media generally have a minimum value of the liquid saturation $S_{l}$, below which the relative permeability of the liquid phase is zero. If the water saturation in the matrix blocks is at this irreducible level, then only the vapor phase (steam) will be mobile. However, although the water cannot flow out of the matrix blocks in the liquid state, water can be produced from the blocks by first vaporizing into the (mobile) steam phase. This is believed to be the case within vapor-dominated geothermal systems, where only steam is produced at the wells (Pruess and Narasimhan, 1982). In such cases, the flow can still be modeled by a single $d$ 'fusion equation. This requires modifying the compressibility term in the diffusion equation to account for the phase change.
Grant and Sorey (1979) derived an expression for the effective compressibility of the water/steam mixture, based on the following analysis. Imagine that the pressure increases, in which case the temperature will also increase, since the water and steam remain in thermodynamic equilibrium. Heat will then flow from the fluid into the rock matrix, causing some of the vapor to condense. Since liquid water is denser than steam, the overall volume of fluid will decrease, giving rise to an apparent compressibility effect. The resulting expression for the effective compressibility is

$(\phi \beta)_{e f f}=\frac{\langle\rho C\rangle T v_{l v}^{2}}{h_{l v}^{2}}$,

where $v_{l v}=v_{v}-v_{l}$ is the difference between the specific volume of the vapor and the liquid, $h_{l v}=h_{v}-h_{l}$ is the latent heat of vaporization, and $\langle\rho C\rangle$ is the overall heat capacity of the system, which is given by

$\langle\rho C\rangle=(1-\phi) \rho_{r} C_{r}+\phi S_{v} \rho_{v} C_{v}+\phi\left(1-S_{v}\right) \rho_{l} C_{l}$,

where $C$ is the specific heat, $\rho$ is the density, and the subscripts $r, l, v$ denote rock, liquid and vapor. This apparent compressibility is usually at least an order of magnitude greater than that of single-phase water or steam. Since the diffusivity is inversely proportional to $(\phi \beta)$, diffusive processes involving two-phase mixtures will proceed relatively slowly.

When the liquid phase is immobile, the flow of the vapor is therefore governed by an equation analogous to equation (2), with the following modifications. The compressibility $(\phi \beta)$ must be replaced by the expression in equation (10), $k_{m}$ must be multiplied by the relative permeability of the rock to the vapor phase, $k_{r v}$, and the viscosity must be taken as that of the vapor, $\mu_{v}$. The relative permeability $k_{r v}$ will vary with vapor saturation, the viscosity $\mu_{\nu}$ will vary with pressure, and $(\phi \beta)_{\text {eff }}$ will vary with both saturation and pressure. In the context of the lumped parameter approximation, we evaluate these parameters at pressure $\bar{P}_{m}$ and $\bar{S}_{\nu}$, where $\bar{S}_{\nu}$ is the mean vapor saturation in the matrix block. During a numerical simulation, these parameters can be reevaluated at each time step, as $\bar{P}_{m}$ and $\bar{S}_{v}$ change with time.

\section{DUAL-POROSITY SIMULATOR}

Numerical reservoir simulators used for porous-medium reservoirs typically solve equation (1), and analogous equations for energy balance, etc., by first discretizing the reservoir into a number of computational gridblocks. A numerical scheme such as finite-differences (Huyakorn and Pinder, 1983), finite elements (Pinder and Gray, 1977), or integral finite-differences (Edwards, 
1972), is then used to reduce the partial differential equations to a set of algebraic equations. These algebraic equations are solved at each time-step $t_{n}$, in order to yield the pressures, temperatures, saturations, etc., in each gridblock at time-step $t_{n+1}=t_{n}+\Delta t$.

We have implemented our semi-analytical dual-porosity model as a modification to the TOUGH simulator (Pruess, 1987), an integral-finite-difference code that has been widely used to simulate the behavior of geothermal reservoirs. The fracture/matrix interaction equations have been incorporated as an option in the source/sink subroutine that is normally used to represent injection or withdrawal of fluid from a well, etc. In our dualporosity simulations, each computational gridblock represents a region that has properties corresponding to the fractured continuum, such as $k_{f}$, $\phi_{f}$, etc., averaged over a suitably-large REV. Each gridblock will also have associated with it three new variables, $\bar{P}, \bar{T}$ and $\bar{S}_{v}$, that represent the (average) thermodynamic state of the fluid in those matrix blocks that are located within that gridblock. Here we drop the subscript $m$ used to denote "matrix", since the overbar serves the purpose of distinguishing the matrix variables from fracture variables. We calculate the pressure change in the matrix blocks, at each time step and for each gridblock, from equation (9), modified as described above:

$(\Phi \beta)_{e f f} \frac{d \bar{P}}{d t}=\frac{\alpha k_{m} k_{r v}\left[\left(P_{f}-P_{i}\right)^{2}-\left(\bar{P}-P_{i}\right)^{2}\right]}{2 \mu_{v}\left(\bar{P}-P_{i}\right)}$,

where $(\phi \beta)_{e f f}$ is found from equation (10), and $\alpha=\pi^{2} / a_{m}^{2}$ (if the matrix blocks are assumed to be spheres of radius $a_{m}$ ). The term on the right-hand side of equation (12) then represents the volumetric fluid flux $Q$, as shown by equation (6). The integration of equation (12) must be done implicitly, in order to avoid numerical instabilities. This means that in passing from $t_{n}$ to $t_{n+1}$, the terms on the right-hand side of equation (12) are evaluated under the conditions that exist at time $t_{n+1}$. An iterative process is therefore required for convergence to the correct new values (see Pruess, 1987). The new saturation at time $t_{n+1}$ is found by applying a mass balance to the matrix block. The mass flux of vapor out of the matrix block into the fracture network also carries with it a sensible heat flux of the amount $\rho_{v} h_{v} Q$, where $Q$ is the volumetric flux. This term must be included in the energy balance equation.

\section{EXAMPLE OF RESERVOIR SIMULATIONS}

To test the accuracy and computational efficiency of the above-described modifications to the TOUGH code, we have simulated some problems using, with some modifications, the computational grid and reservoir properties that were proposed by Spivak (1991) to test geothermal simulators. This is a three-dimensional model of a vapor-dominated geothermal reservoir, with

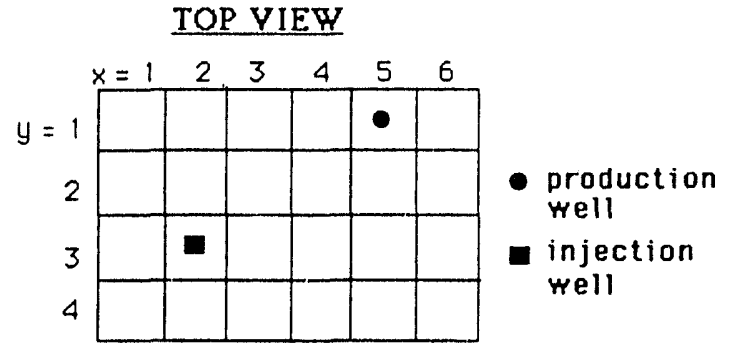

SIDE VIEW

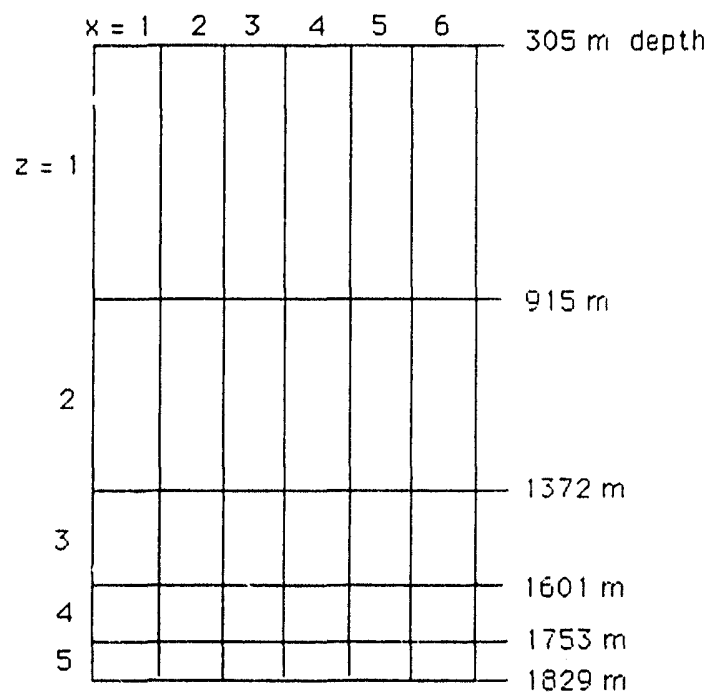

Fig. 1. Schematic diagram of the grid used in simulations of a hypothetical geothermal reservoir. Dimensions of the gridblocks, and the physical properties of the fractures and the matrix blocks, are listed in the text.

properties corresponding to those believed to be applicable to The Geysers geothermal field in California. This hypothetical reservoir (see Fig. 1) is $1524 \mathrm{~m}$ thick, and extends from a depth of $305 \mathrm{~m}$ to $1829 \mathrm{~m}$ below the surface. The cross-sectional shape in any horizontal plane is a rectangle with sides of $914.4 \mathrm{~m}$ and $609.6 \mathrm{~m}$. Each layer is broken up into 24 gridblocks, each of length $152.4 \mathrm{~m}$ in the two horizontal directions. The thicknesses of the five layers are as shown in Fig. 1. A production well (Well \#1) and an injection well (Well $\# 2$ ) are located in gridblocks $x y z=511$ and $x y z=231$ (see Fig. 1), and are completed only in the topmost layer of the reservoir.

The matrix blocks are cuives of $67 \mathrm{~m}$ on each side, with matrix permeability $k_{m}=i \times 10^{-19} \mathrm{~m}^{2}$, matrix porosity $\phi_{m}=0.04$. (A somewhat liow matrix permeability is used so as to avoid having the liquid saturation rise above its irreducible value nftar the injection well). The 
rock has density $\rho_{r}=2648 \mathrm{~kg} / \mathrm{m}^{3}$, and heat capacity $C_{r}=1000 \mathrm{~J} / \mathrm{kg} \mathrm{K}$. The fracture network has an overall porosity $\phi_{f}=0.01$, and permeability $k_{f}=2.0 \times 10^{-14} \mathrm{~m}^{2}$. The relative permeabilities of both the fracture network and matrix blocks are taken to be linear functions of saturation, with the irreducible saturations for the liquid phase, and for the vapor phase in the matrix blocks, set to zero. The irreducible saturation for the liquid in the matrix blocks is 0.25 . In this example, capillary pressure effects and thermal conductivity effects are neglected in both the fractures and matrix blocks.

The initial conditions are that the liquid saturation in the matrix blocks is at its irreducible value of 0.25 , and the pressure in the uppermost layer is $3.45 \mathrm{MPa}$. The initial temperature in the uppermost layer is therefore equal to the saturation temperature at this pressure, which is 242 C. All outer boundaries of the reservoir are impermeable to fluid flow, and the lateral boundaries are also impermeable to heat conduction. A heat flux of $0.5 \mathrm{~W} / \mathrm{m}^{2}$ is conducted vertically upwards through the reservoir. The remaining initial conditions, such as the pressures in the lower layers and the saturations in the fractures, are found by running a simulation to steady state, with no injection or production from the wells.

In the sample problem whose results are shown in Figs. 2.4 , Well \#1 produces $5 \mathrm{~kg} / \mathrm{s}$ of fluid, and Well \#2 injects $5 \mathrm{~kg} / \mathrm{s}$ of liquid water at $95 \mathrm{C}$. The vapor saturation and pressure for the fractures in gridblock 231 are shown in Figs. 2 and 3, respectively, for elapsed times from $10^{1}-10^{8} \mathrm{~s}$ (about 38 months). The solid lines denote the values computed using TOUGH with the modifications described above, whereas the open circles denote values computed with TOUGH using the MINC



Fig. 2. Vapor saturation in the fractures of gridblock 231 , for the problem described in the text.

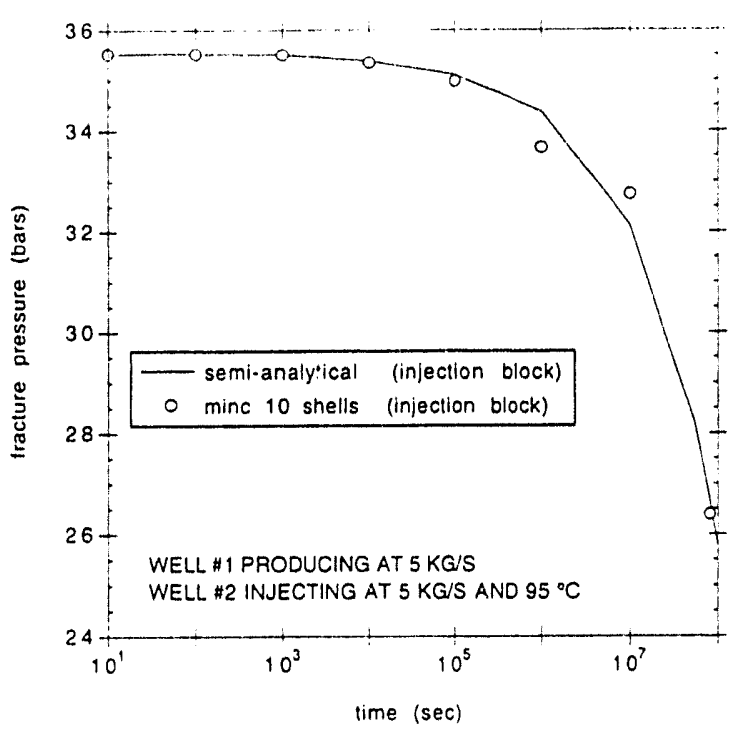

Fig. 3. Pressure in the fractures of gridblock 231, for the problem described in the text.

method to discretize each equivalent matrix block into ten concentric gridblocks. The fracture pressure in gridblock 511 is shown in Fig. 4. The vapor saturation in the fractures in gridblock 511 remains very close to $100 \%$ through both simulations, and is not shown. The predictions of the new method are in all cases very close to those of the MINC simulations. Due to the relatively complex geometry of this problem, and the physical nonlinearities arising from phase-changes, etc., no analytical solution is available for comparison.

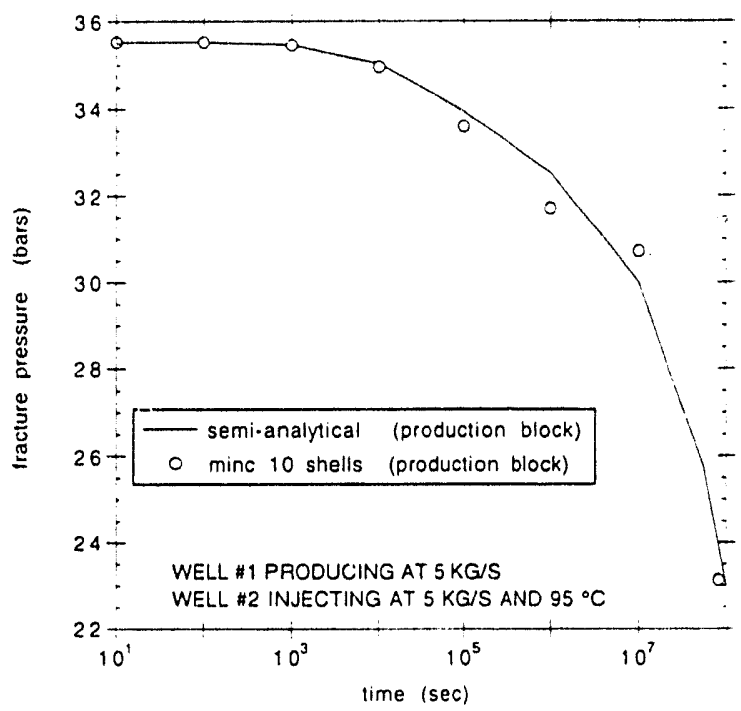

Fig. 4. Pressure in the fractures of gridblock 511, for the problem described in the text. 


\section{CONCLUSIONS}

A new type of dual-porosity model is being developed for two-phase flow processes in fractured geothermal reservoirs. At this time, the model is limited by the assumption that the liquid phase in the matrix blocks remains immobile. By utilizing the effective compressibility concept developed for water/steam mixtures in porous rocks (Grant and Sorey, 1979), flow within the matrix blocks can be modeled by a single diffusion equation. This equation is in turn replaced by a nonlinear ordinary differential equation that utilizes the mean pressure and mean saturation in the matrix blocks to find the rate of fluid flow between the matrix blocks and fractures. This equation has been incorporated into the numerical simulator TOUGH (Pruess, 1987), as a source/sink term for computational gridblocks that represent the fracture system. The accuracy of this new method has been tested by simulating a threedimensional reservoir containing partially-penetrating injection and production wells, and comparing the results to simulations in which the matrix blocks are each discretized into ten concentric shells.

\section{ACKNOWLEDGMENTS}

This work was supported by the Assistant Secretary for Conservation and Renewable Energy, Geothermal Division, U.S. Department of Energy, under Contract No. DE-AC03-76SF00098, with the Lawrence Berkeley Laboratory. The authors thank C. H. Lai and Emilio Antunez of LBL for reviewing this paper.

\section{REFERENCES}

Barenblatt, G. E., Zheltov, Y. P., and Kochina, I. N. (1960), "Basic concepts in the theory of seepage of homogeneous liquids in fissured rocks," J. Appl. Math. Mech., 24, 1286-1303.

Bear, J. (1972), Dynamics of Fluids in Porous Media, Elsevier, New York.

deSwaan, A. (1990), "Influence of shape and skin of matrix-rock blocks on pressure transients in fractured reservoirs," SPE Form. Eval., 5, 344-352.

Duguid, J. O., and Lee, P. C. Y. (1977), "Flow in fractured porous media," Water Resour. Res., 13, 558-566.

Dykhuizen, R. C. (1990), "A new coupling term for dual-porosity models," Water Resour. Res., 26, 351356.

Edwards, A. L. (1972), "TRUMP: A computer program for transient and steady state temperature distribution in multidimensional systems," Rep. UCRL-14754, Lawrence Livermore Laboratory, Livermore, Calif.
Grant, M. A., and Sorey, M. L. (1979), "The compressibility and hydraulic diffusivity of a water-steam flow," Water Resour. Res., 15, 684-686.

Huyakorn, P. S., and Pinder, G. F. (1983), Computational Methods in Subsurface Flow, Academic Press, San Diego, Calif.

Long, J. C. S., and Witherspoon, P. A. (1985), "The relationship of the degree of interconnection to permeability in fracture networks," J. Geophys. Res., 90, 3087-3098.

Matthews, C. S., and Russell, D. G. (1967), Pressure Buildup and Flow Tests in Wells, Society of Petroleum Engineers, Dallas.

Moench, A. F. (1984), "Double-porosity models for a fissured groundwater reservoir with fracture skin," Water Resour. Res., 20, 831-846.

Pinder, G. F., and Gray, W. G. (1977), Finite Element Simulation in Surface and Subsurface Hydrology, Academic Press, San Diego, Calif.

Pruess, K. (1987), "TOUGH User's Guide," Rep. LBL-20700, Lawrence Berkeley Laboratory, Berkeley, Calif.

Pruess, K., and Narasimhan, T. N. (1982), "On fluid reserves and the production of superheated steam from fractured, vapor-dominated geothermal reservoirs," $J$. Geophys. Res., 25, 9329-9339.

Pruess, K., and Narasimhan, T. N. (1985), "A practical method for modeling heat and fluid flow in fractured porous media," SPE J., 25, 14-26.

Pruess, K. P., and Wu, Y.-S. (1989), "A new semianalytical method for numerical simulation of fluid and heat flow in fractured reservoirs," Paper SPE-18426, Society of Petroleum Engineers, Dallas.

Spivak, A. (1991), "Evaluation of Unocal and LBL models for predicting performance of the Geysers geothermal reservoir," Intera West, Los Angeles, Calif.

Streltsova, T. D. (1983), "Well pressure behavior of a naturally fractured reservoir," SPE J., 23, 769-780.

Vermeulen, T. (1953), "Theory of irreversible and constant-pattern solid diffusion," Ind. Eng. Chem., 45, 1664-1670.

Warren, J. E., and Root, P. J. (1963), "The behavior of naturally fractured reservoirs," $S P E J ., 3,245-255$.

Zimmerman, R. W., Chen, G., and Bodvarsson, G. S. (1992), "A dual-porosity reservoir model with an improved coupling term," 17th Workshop on Geother. mal Reservoir Engineering, Stanford, Calif. 